\title{
Solide Nabelschnurtumoren.
}

Von

Rudolf Herweg in Lüneburg.

(Hierzu Tafel VIII-IX.)

Bei der Seltenheit solider Nabelschnurtumoren - nach von Winckel sind bisher nur fünf Fälle bekannt geworden halte ich es für angebracht, einen vor kurzem von mir beobachteten Fall zu veröffentlichen. Dieser Nabelschnurtumor nimmt den bisher beschriebenen gegenüber insofern eine Sonderstellung ein, als er sich nahe der Placenta, dicht vor der Insertion der Nabelschnur, fand; in den früheren Fällen sass die Geschwulst am Nabelring oder sie stand zu Nabelhernien in Beziehung. Auch hinsichtlich des Ausgangspunktes weicht der vorliegende Tumor von den bisher bekannten ab. Während letztere zu den von der Bauchhaut aus in den persistirenden Theil des Nabelstranges hineinragenden Capillaren vielleicht auch zu den nach Ruge in einzelnen Fällen im Nabelstrang vorkommenden grossen Capillarnetzen der persistirenden Vasa omphalo-mesaraica in Beziehung standen, befindet sich die von mir beobachtete Geschwulst, wie wir später sehen werden, in Zusammenhang mit einer der beiden Umbilicalarterien.

Neben dem eigentlichen soliden Tumor finden sich noch vier sackartige Anhänge. Eines dieser Gebilde sitzt breitbasig neben dem Tumor der Nabelschnur auf, drei sind mehr kugelig blasenartig. Die grösste Blase ist kirschgross, die zweite kirschkerngross, die dritte etwa hanfkorngross. Diese Anhänge und sulzreiche Windungen der einen Nabelschnurarterie lassen das abnorme Nabelschnurgebilde auf den ersten Blick recht complicirt erscheinen.

Die Abbildung 1 (Taf. VIII) zeigt ein Stück der Placenta mit Nabelschnurinsertion, den Tumor, die blasenartigen Anhänge und die sulzreiche Nabelarterienwindung. Von dem soliden Tumor, der Insertionsstelle und dem angreuzenden Placentartheil ist die Amnionhülle abgelöst und zur Seite geschlagen. Diese Hülle ist unten 
mit einem schmalen Streifen mit dem Tumor in Verbindung geJassen, was Abbildung 2 (Taf. VIII) besonders veranschaulicht.

Die Gestalt des soliden Tumors ist nierenförmig. Consistenz und Aussehen im Innern ist einem Fibromyom vergleichlich. Die Länge der Geschwuist beträgt $5 \mathrm{~cm}$, die Höhe $3,3 \mathrm{~cm}$ und die Breite $3,2 \mathrm{~cm}$. Die äussere Umhüllung bildet eine weichere $1 / 2$ bis $1 \mathrm{~cm}$ breite Schicht, die das Aussehen und die Consistenz der Nabelschnursulze besitzt und mit Amnion überzogen war. Die Abbildung des bis auf die Basis durchschnittenen Tumors (Abb. 2, Taf. VIII) lässt stellenweise die Grenze zwischen Sulzschicht und Geschwulstmasse deatlich erkennen. Die Schnittfläche des Tumors zeigt verkalkte Partien, grössere und kleinere, quer und schräg getroffene Blutgefässe. Eine Untersuchung des Verlaufes der Nabelschnurgefässe und ihr Verhalten zum Tumor hatte folgendes interessante Ergebniss. An der Insertionsstelle auf der Placenta münden zwei arterielle Gefässe neben der Nabelschnurvene.

Ein drittes arterielles Gefäss tritt etwa $1 \frac{1}{2} \mathrm{~cm}$ entfernt in die Placenta ein. Da letzterwähnte Arterie abseits von den übrigen Gefässen auf dem Mutterkuchen inserirt, wurde angenommen, dass man es mit einer von einer Nabelschnurärterie entspringenden Verzweigung zu thun hätte. Um dies festzustellen, wurde mittelst Sonde und Scheere dieses Gefäss verfolgt und sein Lumen eröffnet und zwar von der Placentarinsertion ausgehend. Als erstes fand sich an der Insertionsstelle eine Stenose, dicht oberhalb dieser Stelle, d. h. fötalwärts, eine erbsengrosse starrwandige aneurysmatische Erweiterung. Die Arterie blieb bis auf Weiteres oberhalb des Aneurysmas dilatirt und verlief durch die sulzige Substanz an der Basis des Tumors in S-förmiger Biegung aufsteigend. Mitten unter der Geschwulst wurde ein Gefässast in den Tumor abgegeben, der sich in der Geschwuist vielfach verzweigte.

Thren weiteren Verlauf nahm die Arterie dann in der sulzigen Masse unter den blasenartigen Anhängen hindurch, bildete in der in Abb. 1 bezeichneten Partie eine von Sulze umgebene Schleife und liess sich nun als die eine der Nabelschnurarterien bis zu der Nabelschnurschnittstelle verfolgen. Hieraus erhellt, dass es sich um einen echten, von einer Nabelschnurarterie ernährten, Nabelschnurtumor handelt. Ausser dem den Tumor versorgenden Gefässast ist an der eben beschriebenen Nabelarterie kein Gefässabgang mehr beobachtet worden, demnach müssen die beiden obengenannten neben der Vene inserirenden arteriellen Gefässe der andern Nabel- 
schnurarterie angehören. - Die Nabelschnur zeigte im Uebrigen keinerlei Abweichungen, sie hatte eine Länge von $54 \mathrm{~cm}$, war nicht abnorm gedreht und nicht um Kindstheile geschlungen gewesen.

Zur Artbestimmung des Tumors untersuchte ich ein von der Halbirungsschnittfläche entnommenes Stück, welches die äussere sulzige Hülle neben Tumorgewebe enthielt, mikroskopisch: Abbildung 3 (Taf. IX). Die sulzige Aussenschicht wird durch eine gefässlose, homogene (schleimige) Substanz gebildet, welche mit zahlreichen, feinen, welligen Fasern maschenartig durchzogen ist: Die am meisten hervortretenden Fasern weisen spindelige und stäbchenförmige Kerne auf, vereinzelt finden sich auch dreizipflige sogenannte sternförmige Zellen mit ovalem Kern, deren faserige Ausläufer ununterbrochen ineinander übergehen. Sind diese Zellen auch nur spärlich vorhanden, so glaube ich doch, auf Grund dieses Befundes annehmen zu müssen, dass man es in der Aussenschicht des Tumors mit Warthon'scher Sulze zu thun hat. Der im Gegensatz zu normaler Sulze lockere Faserbau und das geringe Vorhandensein von kernhaltigen Zellen würde ja durch die Dehnung, die die Sulzschicht durch die Expansion der Geschwulst erfahren hat, ausreichend erklärt sein.

Die eigentliche Geschwulstmasse wird aus einer der Aussenschicht mikroskopisch gleichenden Grundsubstanz gebildet, die aber von zahllosen Gefässen durchsetzt ist. Das Lumen und die Gestalt dieser Gefässe ist sehr verschieden. Viele quergeschnittene zeigen Ringform von kleinster bis zu ganz erheblicher lichter Weite. Andere wieder sind oval oder bilden kleinere und grössere seitliche Verzweigungen und Anastomosen. Alle diese Gefässe sind mit Endothel ausgekleidet. Thre Wandungen haben versehiedene Stärke, Muskelzellen umgeben das Endothel in ein, zwei, drei und mehr Lagen. Häufig findet sich besonders bei den Gefässen mit weitestem Lumen nur Endothelwand. Während es sich in ersteren um arterielle Gefässverästelungen und Capillaren handelt, sind letztere als erweiterte Capillaren anzusehen. In einigen wenigen Gefässen finden sich auch noch rothe Blutkörperchen vor. Auf Grund dieses Befundes liegt ohne Frage ein "Angiom" vor. Da das Grundgewebe aus schleimiger spindel- und sternzellenhaltiger Substanz gebildet wird, dürfte der Tumor genauer ein "Hyxangiom" genannt werden.

Herr Professor Kaufmann-Göttingen, den ich um die Begutachtung des mikroskopischen Präparates der soliden Geschwulst gebeten habe, theilte mir mit, es handle sich um einen angiomatös- 
myxomatösen Tumor, bei den Verkalkungen um verkalkte Thromben, theilweise auch Gefässwände. Es sei mir an dieser Stelle gestattet, Herrn Professor E. Kaufmann für den mir gütigst ertheilten Bericht seiner mikroskopischen Untersuchung meinen verbindlichsten Dank auszusprechen. -

Auf die blasenartigen eystenähnlichen Anhänge, die nahe der Geschwulst auf der Sulze ihren Sitz haben, möchte ich nur kurz eingehen. Die kirschgrosse Blase habe ich eröffnet. Sie enthielt eine dünnschleimige, fast klare Flüssigkeit. Im Innern fand sich ein mit unbewaffnetem Auge sichtbares, milehig trübes Balkengeflecht. Beim Herausschneiden eines breiten Stückes collabirten die Balkenräume mehr und mehr. Im mikroskopischen Bild sieht man aussen den Ueberzug des Amnionepithels, darunter ein sehr kernarmes Maschennetz aus feinen welligen Faserbündeln mit spindeligen und sternförmigen Zellen, ähnlich der oben beschriebenen Sulzschicht des Tumors. Ejgenthümlich ist hier nur, dass die Spalträume nach dem Centrum der Blase hin an Grösse zunehmen. Ein einheitlicher Cystensack mit Epi- oder Endothelauskleidung war nicht vorhanden. Ich sehe nach diesem. Bilde die blasenartigen Anhänge als eine durch den Reiz des Myxangioms oder durch Cireulationsstörung ausgelöste myxomatöse Veränderung der Warthonschen Sulze an, es handelt sich hier aber nicht um Cysten der Nabelschnur, wie sie beispielsweise von Fava und Panciera, Hahn u. A beobachtet worden sind.

Was nun die andern bisher beobachteten soliden Tumoren des Nabelstranges anlangt, so sind die zwei ersten Fälle nicht genauer histologisch untersucht worden. Der erste von Maunoir 1820 gesehene Tumor bestand in einer erdbeergrossen, fungösen Geschwulstmasse, welche auf der Kuppe einer Nabelschnurhernie sass.

Den zweiten Fall, eine birnengrosse Geschwulst mit teleangiectatischem Bau an der Basis einer Nabelschnurhernie beobachtete Lawton.

Die drei letztgefundenen soliden Nabelschnurtumoren, die genau histologisch untersucht worden sind, waren teleangiectatische Myxosarkome.

Den einen fand Gerdes. Fin vier Zoll langer, zeigefingerdicker, kuhhornförmiger Tumor drängte sich als hochrothe, ziemlich feste Geschwulst mit dem Nabelstrang aus dem Nabelring.

Der von Lissner operirte und von Kaufmann untersuchte 
Tumor entsprang als $6 \mathrm{~cm}$ hohe und $16 \mathrm{~cm}$ an der Peripherie messende Geschwulst im Nabelring.

Der fünfte von. v. Winckel in der Sammlung klin. Vorträge, No. 140, 1895 genau beschriebene Tumor sass als hochrothe, ziemlich derbe, unregelmässig geformte Neubildung von $4 \mathrm{~cm}$ Länge gleichfalls im Nabelring neben der Nabelschnur.

Wie eingangs erwähnt, sind als Ausgangspunkt der bisher bekannt gewesenen soliden Nabelschnurgeschwülste, die von der Bauchbaut aus in den persistirenden Theil des Nabelstranges hineinragenden Capillaren angesehen. Als Ausgangspunkt der von mir beobachteten Geschwulst muss die eine der beiden Nabelschnurarterien gelten, geht doch von ihr aus ein viel verzweigter Gefässast in den Tumor hinein.

Als Ursache der vorliegenden Neubildung ist meines Erachtens eine Circulationsstörung des Endabschnittes der Umbilicalarterie anzusehen. Wie oben beschrieben, fndet sich an ihrer Inscrtionsstelle an der Placenta eine deutliche Verengung und oberhalb dieser Stenose eine aneurysmatische Erweiterung mit bis über den Tumor hinaus zu verfolgender Dilatation der Arteria umbilicalis. In der durch die grobanatomischen Verhältnisse: Stenose, Aneurysma und Dilatation der Arteria umbilicalis bedingten über jeden Zweifel erhabenen Circulationsstörung möchte ich einen Beweis sehen für die Annahme von Winckel's, dass bei der Entstehung congenitaler Geschwülste Circulationshindernisse (und Traumen) eine Rolle spielen.

Zam Schluss sei der Geburtsbericht angefügt, da er noch manches Interessante bietet.

Am 24. Januar a. c. wurde ich zu der 26 Jahre alten Kreissenden, Frau M. L., gerufen und zwar auf Veranlassung der Hebamme, weil die Gebärende während ihrer Gravidität im October 1908 eine fieberhafte Lungenerkrankung durchgemacht hatte und noch sehr schwach war.

Wie mir der seinerzeit behandelnde College berichtete, hatte es sich um linksseitige Tuberculose suspecte Pleuritis exsudativa gehandelt. Der Erguss bildete sich ohne Punction zuräck. Fieber hatte bis $39^{\circ}$ bestanden. Patientin erholte sich leidlich.

Frau L. stammt von gesunden Eltern und besitzt drei gesunde Geschwister. In der Familie des gesunden kräftigen Mannes sind angeblich keine erblichen Krankheiten yorgekommen. Die Gebarende bat als Kind von 10 Jahren Lungenentzündung gehabt, ist aber sonst bis zum letzten Herbst nicht ernstlich krank gewesen. Sie neigt nur bei den leichtesten Erkältungen zu Husten. Auswurf ist dabei stets spärlich, nie blutig.

Die Periode trat seit ihrem Beginn immer regelmässig in vierwöchentlichen Intervallen auf, war von fünftägiger Dauer mit nur geringen Blutverlusten. Am 15. September 1904 spontaner Partus eines bis heute 
gesunden kräftigen Mädchens. Das Wochenbett verlief ungestört. Das Kind wurde ein Jahr lang gestillt.

Im August 1907 gebar Patientin nach achtwöchentlichem Ausbleiben der Periode unter starken Blutverlusten mit ärztlicher Hülfe eine Blasenmole. Nach fieberfreiem Heilungsverlaufe erholte sie sich schnell und fühlte sich ganz gesund.

Am 19. April 1908 war die letate Regel vor der diesjährigen Geburt. Der Partus ging trotz der Schwäche der Frau gut von statten. Ich kam in der Autreibungszeit hinzu und fand die Frucht in erster vollkommener Fusslage. Der Allgemeinzustand der Kreissenden war, abgesehen von der Blässe und dem mangelhaften Ernährungszustand ein derart guter, dass ich erst eingriff; als die Frucht bis über den Nabel geboren war. Die Wehen waren dauernd gut. Das Kind weiblichen Geschlechts wurde lebensfrisch geboren, es zeigte die Zeichen der Reife, seine Länge betrug $49 \mathrm{~cm}$. Eine halbe Stunde nach der Geburt des Kindes wurde die gelöste Nachgeburt exprimirt, die Placenta war vollständig mit Eihäuten. Die $54 \mathrm{~cm}$ lange Nabelschnur bot bis anf die Veränderungen im Bereich des Tumors nichts Bemerkenswerthes.

Das Wochenbett rerlief fieberfrei. Wegen wunder Warzen und Nahrungsmangels musste das Kind bald abgesetzt werden. Das Neugeborene erkrankte an Pemphigus neonatorum, ist aber nachdem, wie ich mich Fnde Mai überzeugt habe, gut gediehen.

\section{L i teratur.}

Seitz, L., Erkranikungen der Eihänte. Handbuch der Geburtshülfe von v. Winckel. II. Bd. 2.

Seitz, L., Placentartumoren, Choriangiome. Handbuch der Geburtshülfe yon v. Winckel. II. Bd. 2.

Stoeckel, W., Geburtsstörungen infolge von Anomalien der Eihäute und der Nabelschnur. Handbueh der Geburtshülfe von v. Winckel. II. Bd. 3. Stroeckel, W., Geschwulstbildungen des Nabelstranges. Handbuch der Geburtshülfe von $\mathrm{v}$. Winckel. II. Bd. 3.

v. Winckel, F., Ueber angeborene solide Gesohwülste des perennirenden Theiles der Nabelschnur. Samml. klin. Vortr. No. 140. Leipzig 1895.

Kaufmann, E., Veber eine Geschwulstbildung des Nabelstranges. Virchow's Archiv. 1890. Bd. 121.

Hahn, C. F. O., Ueber ein cystenartiges Gebilde im Nabelstrang einer Traubenmole. Inaug.-Dissert. Leipzig 1864.

\section{Erklärungen der Abbildungen auf Tafel VIII und IX.}

Abbildung 1 und 2. Photographische Aufnahmen. Atelier Gustar Nause, Lüneburg.

Abbildung 3. Gezeichnet von der Zeichnerin im Königl. Patholog. Institut zu Göttingen E. R. Bieling: 


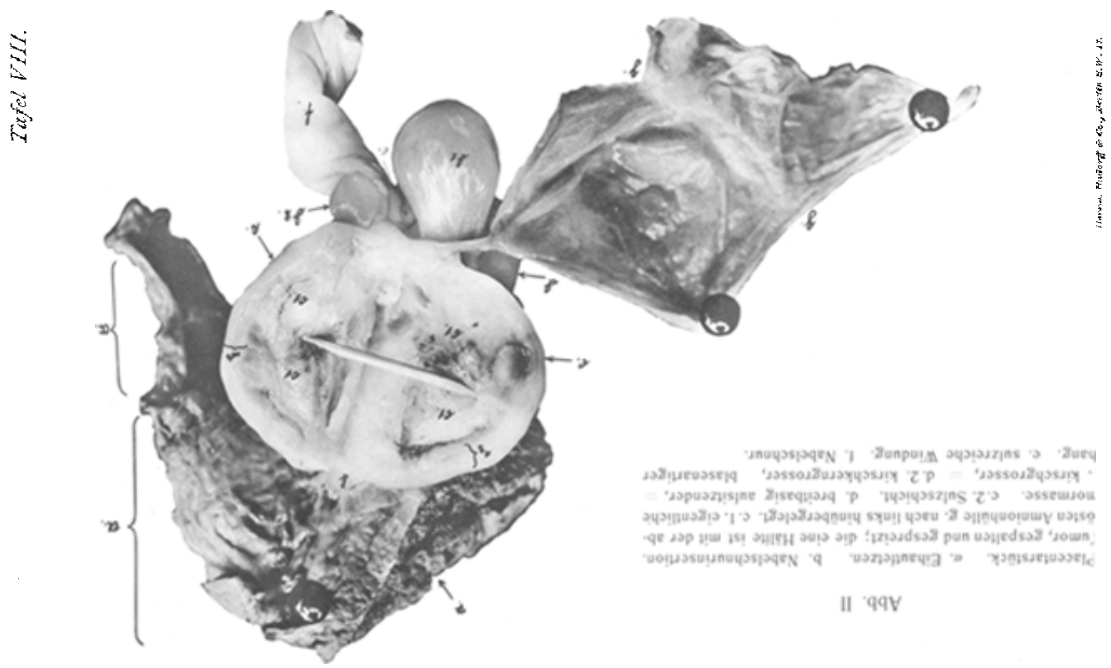

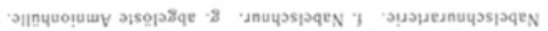

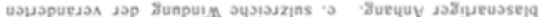

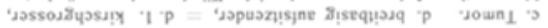

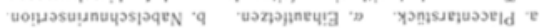



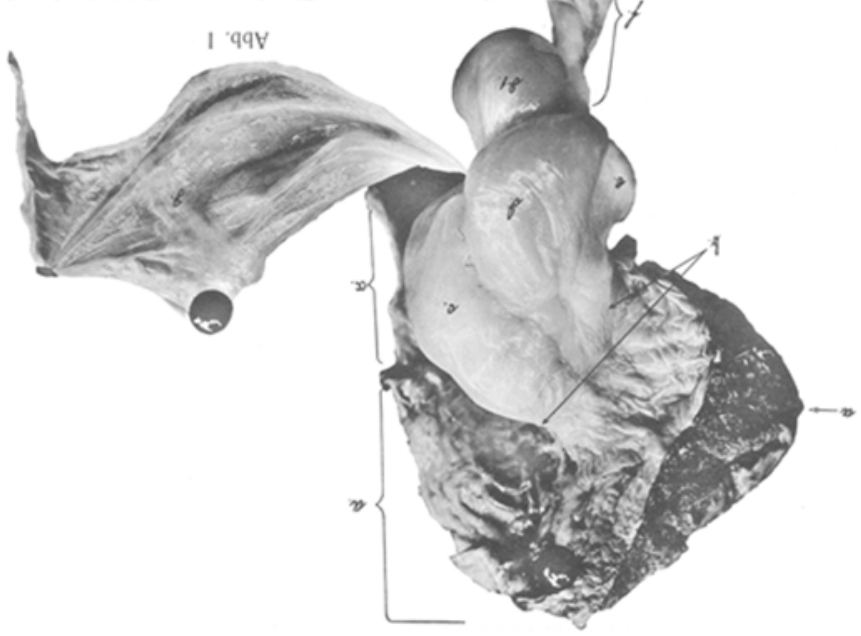




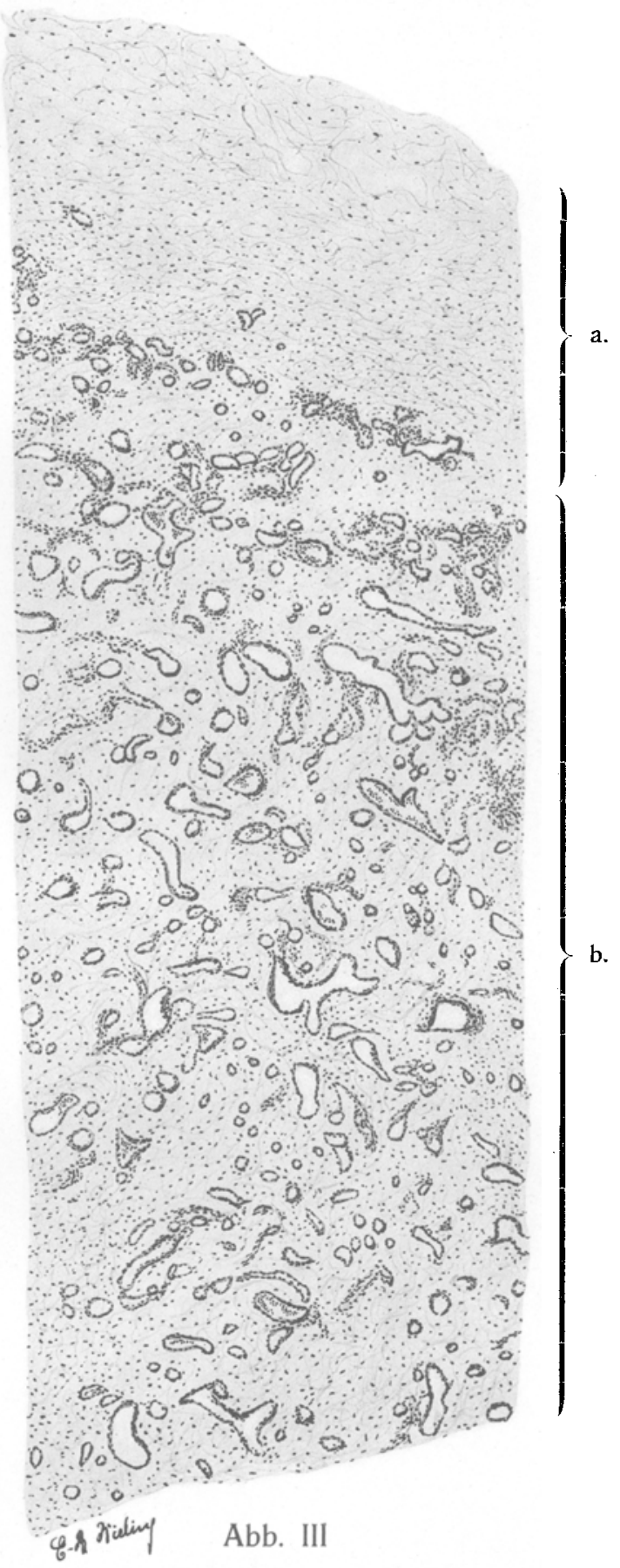

a. die den Tumor rings umgebende Sulzschicht. b. angiomatösmyxomatöse Tumorsubstanz. 